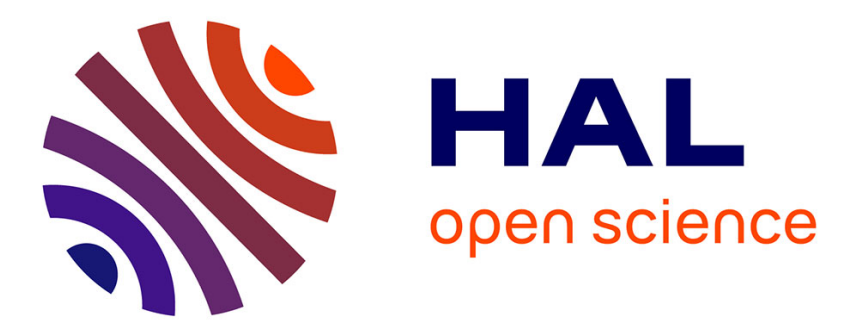

\title{
Optimal Power Allocation for Minimizing the Energy Consumption of a NOMA Base Station with Cell DTx
}

\author{
Rémi Bonnefoi, Haïfa Farès, Pierre Bélis, Yves Louet
}

\section{To cite this version:}

Rémi Bonnefoi, Haïfa Farès, Pierre Bélis, Yves Louet. Optimal Power Allocation for Minimizing the Energy Consumption of a NOMA Base Station with Cell DTx. URSI AP-RASC, Mar 2019, New-Delhi, India. 10.23919/ursiap-rasc.2019.8738765 . hal-01948638

\section{HAL Id: hal-01948638 \\ https://hal.science/hal-01948638}

Submitted on 7 Dec 2018

HAL is a multi-disciplinary open access archive for the deposit and dissemination of scientific research documents, whether they are published or not. The documents may come from teaching and research institutions in France or abroad, or from public or private research centers.
L'archive ouverte pluridisciplinaire HAL, est destinée au dépôt et à la diffusion de documents scientifiques de niveau recherche, publiés ou non, émanant des établissements d'enseignement et de recherche français ou étrangers, des laboratoires publics ou privés. 


\title{
Optimal Power Allocation for Minimizing the Energy Consumption of a NOMA Base Station with Cell DTx
}

\author{
Rémi Bonnefoi $^{(1)}$, Haïfa Farès $^{(1)}$, Pierre Bélis $^{(1)}$ and Yves Louët ${ }^{(1)}$ \\ (1) CentraleSupélec/IETR, CentraleSupélec Campus de Rennes, 35510 Cesson-Sévigné, France
}

\begin{abstract}
In this paper, we consider a base station that jointly employs Non-Orthogonal Multiple Access (NOMA) and Cell Discontinuous Transmission (Cell DTx) in order to reduce its power consumption and we study the problem of optimal power allocation. We show first that this problem is convex and can be solved using only single variable root finding algorithms. Then, we employ numerical simulations in order to assess the performance of the proposed power allocation.
\end{abstract}

\section{Introduction}

Non-Orthogonal Multiple Access (NOMA) allows to multiplex several users in the power domain [1]. With this multiple access technique, the base station serves users with the same time and frequency resources, using different transmit powers. At the receiver side, the users employ Successive Interference Cancellation (SIC) so as to eliminate successively the interfering signals with the highest transmit power until they access to their data. NOMA is then considered as a promising energy-efficient transmission ensuring massive connectivity objective for $5 \mathrm{G}$ communications.

From another side, Cell Discontinuous Transmission (Cell DTx) [2] is typically used to reduce the energy consumption of a base station by switching into sleep mode during small periods in each frame.

In this paper, we study the problem of power allocation when combining both techniques NOMA with Cell DTx. The problem of power allocation when considering Cell Dtx either with Time Division Multiple Access (TDMA) $[3,4]$ or with Orthogonal Frequency Division Multiple Access (OFDMA) [5, 6] has already been studied. However, as far as the authors knowledge goes, the problem of power allocation with NOMA and Cell DTx has not been studied in the literature yet.

We first set the problem of power allocation with NOMA and Cell DTx. Second, we show that this problem can be written in a convex form and, consequently, its solution can be obtained following the Karush-Kuhn and Tucker (KKT) conditions. Then, we use numerical simulations in order to compare the performances of the optimal NOMA and TDMA power allocations with Cell DTx.
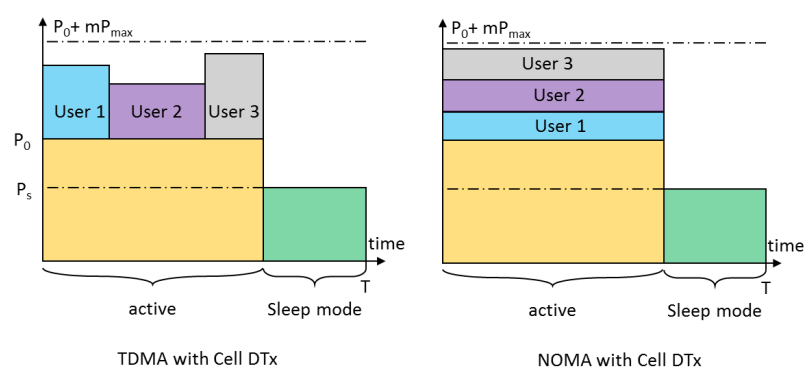

Figure 1. Comparison of the power allocation with NOMA and TDMA with Cell DTx.

The rest of this paper is organized as follows. The system model is introduced in Section II. In Section III, we formulate the problem of power allocation with Cell DTx, we prove its convexity and we propose an algorithm to solve it. Some numerical results are introduced in Section IV. Finally, conclusions are conducted in Section V.

\section{System Model}

We consider a base station that serves $N_{u}$ users with NOMA. We assume flat fading channels and we denote by $g_{k}$ the channel coefficient of user $k$. Moreover, $B$ denotes the bandwidth and $\sigma^{2}$ is the thermal noise variance. We denote by $P_{\mathrm{Tx}}^{k}$ the transmit power of user $k$.

Furthermore, the total power consumption of a base station can be modeled as [7]:

$$
\left\{\begin{array}{cccc}
P_{\text {supply }} & = & P_{0}+m P_{\mathrm{Tx}} & \text { if } 0<P_{\mathrm{Tx}} \leq P_{\max } \\
P_{\text {supply }}= & P_{S} & \text { else }
\end{array}\right.
$$

Where $P_{0}$ is the static power consumption of the base station, $P_{\text {Tx }}$ the RF transmit power which is lower than the maximum RF transmit power of the base station denoted $P_{\max } . m$ is the coefficient of the load dependence and $P_{s}$ the power consumption of the base station during sleep mode.

As illustrated in Figure 1, we suppose that the base station uses NOMA for multiple access. We assume that, during each frame of duration $T$, the base station serve the users using NOMA during $t_{a}$ and then switches to sleep mode in 
order to save energy. Compared to the TDMA power allocation introduced in [3], with NOMA, the users are separated in the power domain when the base station is active. Without loss of generality, we index user in descending order of their channel coefficient, i.e., $g_{1}>g_{2}>\cdots>g_{N_{u}}$. In NOMA, the transmit power of the served users is allocated in reverse order of the channel coefficients, i.e., $P_{\mathrm{Tx}}^{1}<P_{\mathrm{Tx}}^{2}<\cdots<P_{\mathrm{Tx}}^{N_{u}}[8]$.

\section{Problem formulation and resolution}

Our objective is to minimize the base station energy consumption while providing each user a given minimum rate. To do so, we minimize the average power consumption of the base station during each frame. Moreover, we suppose that the rate of each user is equal to the Shannon capacity when the base station is active and equal to zero when the base station switches to sleep mode. Assuming a perfect SIC and denoting $\mu_{a}=\frac{t_{a}}{T}$, the rate of a user during a frame is equal to:

$$
C_{k}=B \mu_{a} \log _{2}\left(1+\frac{P_{\mathrm{Tx}}^{k} g_{k}}{\sigma^{2}+g_{k} \sum_{l=1}^{k-1} P_{\mathrm{Tx}}^{l}}\right) .
$$

Denoting $C^{\min }$ the minimum capacity required by the users served by the base station, the problem of power allocation with NOMA and Cell DTx becomes

$$
\begin{array}{cl}
\underset{\mu_{a}, P_{\mathrm{Tx}}}{\operatorname{argmin}} & \mu_{a}\left(P_{0}+m \sum_{k=1}^{N_{u}} P_{\mathrm{Tx}}^{k}\right)+\left(1-\mu_{a}\right) P_{s}, \\
\text { S.t. } & C_{k} \geq C^{\min }, \quad \forall k, \\
& 0 \leq \mu_{a} \leq 1, \\
& \sum_{k=1}^{N_{u}} P_{\mathrm{Tx}}^{k} \leq P_{\max }, \\
& P_{\mathrm{Tx}}^{k} \geq 0 .
\end{array}
$$

Using the same reformulation as in [9], we introduce the variables $\Gamma_{k}=\sum_{l=1}^{k} P_{\mathrm{Tx}}^{k}$ so as to rewrite the problem in a more tractable form. We obtain:

$$
\begin{gathered}
\underset{\mu_{a}, \Gamma_{N_{u}}}{\operatorname{argmin}} \quad \mu_{a}\left(P_{0}+m \Gamma_{N_{u}}\right)+\left(1-\mu_{a}\right) P_{s}, \\
\text { S.t. } \Gamma_{k} \geq \frac{\sigma^{2}\left(2^{\frac{C^{\min }}{\mu_{a} B}}-1\right)}{g_{k}}+\Gamma_{k-1} 2^{\frac{C^{\min }}{\mu_{a} B}}, \forall k, \\
\Gamma_{1} \geq \frac{\sigma^{2}\left(2^{\frac{C^{\min }}{B \mu_{a}}}-1\right)}{g_{1}}, \\
\Gamma_{N_{u}} \leq P_{\max }, \\
0 \leq \mu_{a} \leq 1 .
\end{gathered}
$$

It is obvious that the power consumption is minimum when the rate of each user is equal to the minimum required rate.
In other words, when the inequality in the capacity constraint becomes an equality. In that case, denoting $\Gamma_{0}=0$, we obtain subsequently the relation between $\Gamma_{k}$ and $\Gamma_{k-1}$ :

$$
\Gamma_{k}=\frac{\sigma^{2}}{g_{k}}\left(2^{\frac{C^{\min }}{B \mu_{a}}}-1\right)+2^{\frac{C^{\min }}{B \mu_{a}}} \Gamma_{k-1}
$$

Starting from $\Gamma_{1}$, after $k$ iterations we can express $\Gamma_{k}$ as a function of $\mu_{a}$ :

$$
\begin{gathered}
\Gamma_{k}=\sigma^{2}\left(2^{\frac{C^{\min }}{B \mu_{a}}}-1\right) \sum_{l=1}^{k-1} \frac{2^{\frac{C^{\min }(k-l)}{B \mu_{a}}}}{g_{l}} \\
+\frac{\sigma^{2}\left(2^{\frac{C^{\min }}{B \mu_{a}}}-1\right)}{g_{k}} .
\end{gathered}
$$

We obtain a new expression for the problem of power allocation that only depends on $\mu_{a}$, the time during which the base station is active:

$$
\begin{aligned}
& \underset{\mu_{a}}{\operatorname{argmin}} \mu_{a} P_{0}+m \mu_{a} \sigma^{2}\left(2^{\frac{C^{\min }}{B \mu_{a}}}-1\right) \sum_{l=1}^{N_{u}} \frac{2^{\frac{C^{\min }\left(N_{u}-l\right)}{B \mu_{a}}}}{g_{l}} \\
& +\left(1-\mu_{a}\right) P_{s} \\
& \text { S.t. } \quad \mu_{a} \sigma^{2}\left(2^{\frac{C^{\min }}{B \mu_{a}}}-1\right) \sum_{l=1}^{N_{u}} \frac{2^{\frac{C^{\min }\left(N_{u}-l\right)}{B \mu_{a}}}}{g_{l}} \leq \mu_{a} P_{\max }, \\
& \quad 0 \leq \mu_{a} \leq 1 .
\end{aligned}
$$

Using the latter reformulation, we are able to compute $\mu_{a}$ so as to solve the problem of power allocation. Our resolution relies on the following proposition.

Proposition 3.1. The problem defined by equation (7) is convex.

Proof. In order to prove the convexity, it is sufficient to prove that the function $g: \mu_{a} \mapsto \mu_{a} 2^{\frac{C^{\min } k}{B \mu_{a}}}\left(2^{\frac{C^{\min }}{B \mu_{a}}}-1\right)$ is convex.

$$
\frac{d^{2} g}{d \mu_{a}^{2}}=2^{\frac{C^{\min } k}{B \mu_{a}}} \frac{C^{\min 2} \log (2)^{2}}{B^{2} \mu_{a}^{3}}\left(2^{\frac{C^{\min }}{B \mu_{a}}}(k+1)^{2}-k^{2}\right) \geq 0 .
$$

As a consequence, $\mathrm{g}$ is convex and so is the objective function of the problem defined by (7), since it is a sum of convex functions. Furthermore, the constraints in (7) are also convex. Consequently, the studied problem is convex.

As the problem is convex, we can apply the Karush-KuhnTucker (KKT) conditions to solve it. With these conditions, each inequality constraint can either be binding (an equality) or non-binding. We can consequently have three cases depending on either constraints are binding or not: 


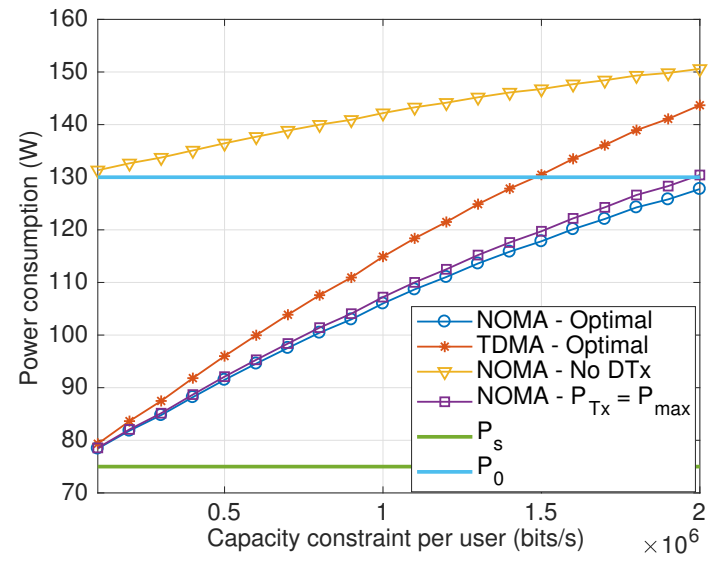

Figure 2. Average base station power consumption as a function of the capacity constraint of the users.

1. Both constraints of the problem defined by (7) are not binding.

2. The transmit power is equal to the maximum transmit power of the base station. In other words, the first constraint of the problem defined by (7) is binding.

3. The total service time is equal to 1. I.e. the base station does not switch to sleep mode and the second constraint of of the problem defined by (7) is binding.

In order to compute $\mu_{a}$, we are first going to solve the problem without considering the constraints. Then, we verify if the constraints are satisfied. If we are not in this case, and according to the KKT conditions, the optimum is obtained when the non-satisfied constraint is binding. Finally, once the value of $\mu_{a}$ is obtained, we compute the transmit powers with equation (6).

Case 1: When none of the constraints is binding, the optimal value of $\mu_{a}$, denoted $\mu_{\mathrm{opt}}$, is the point where the derivative of the objective function is equal to zero. It is the solution of:

$$
\begin{aligned}
& \frac{C^{\min } \log (2)}{B \mu_{a}} \sum_{l=1}^{N_{u}} \frac{2^{\frac{C^{\min }\left(N_{u}-l\right)}{B \mu_{a}}}}{g_{l}}\left(2^{\frac{C^{\min }}{B \mu_{a}}}\left(N_{u}-l+1\right)-\left(N_{u}-l\right)\right)= \\
& \frac{\left(P_{0}-P_{s}\right)}{m \sigma^{2}}+\left(2^{\frac{C^{\min }}{B \mu_{a}}}-1\right) \sum_{l=1}^{N_{u}} \frac{2^{\frac{C^{\min }\left(N_{u}-l\right)}{B \mu_{a}}}}{g_{l}}
\end{aligned}
$$

This equation can be solved using a single variable root finding algorithm

Case 2: In that case, the transmit power is equal to the maximum transmit power of the base station and the optimal value of $\mu_{a}$ is the solution of:

$$
\mu_{a} \sigma^{2}\left(2^{\frac{C^{\min }}{B \mu_{a}}}-1\right) \sum_{l=1}^{N_{u}} \frac{2^{\frac{C^{\min }\left(N_{u}-l\right)}{B \mu_{a}}}}{g_{l}}=\mu_{a} P_{\max }
$$

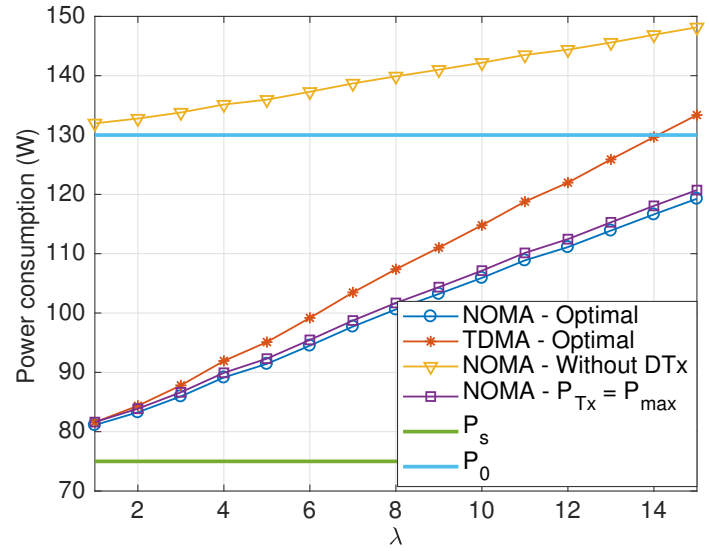

Figure 3. Average base station power consumption as a function of the intensity of the PPP.

Case 3: In that case, the base station does not switch to sleep mode and $\mu_{a}=1$

\section{Numerical results}

In this section, some numerical simulations are given to assess the performances of the proposed optimal power allocation with NOMA and Cell DTx. To do so, we consider a base station that serves $N_{u}$ users over a band of bandwidth $B=10 \mathrm{MHz}$. For this base station, $P_{0}=130 \mathrm{~W}, P_{s}=75$ $\mathrm{W}, m=4.7$ and $P_{\max }=20 \mathrm{~W}$ [7]. The served users are distributed following a Poisson point process, we denote $\lambda$ the average number of users in the cell coverage. Besides the distance between the users and the base station varies between 300 and $1500 \mathrm{~m}$. The pathlosses are computed with the Winner II ' $\mathrm{C} 1$ ' pathloss model. Besides, we consider a $10 \mathrm{dBi}$ of antenna gain and a $2 \mathrm{~dB}$ of noise figure. Furthermore, we consider that the served users have the same capacity constraint.

Fig. 2 depicts the average power consumption of a base station (operating with the DTx mode) as a function of the capacity constraint per user for a fixed average number of users in the cell coverage equal to 10. For seek of comparison, results are given for both TDMA and NOMA techniques. Regardless the target capacity constraint per user, combining NOMA with DTx mode always benefits to the system with respect to TDMA with DTx. This yields to sizable power savings especially for high capacity constraints. This conclusion has already been emphasized for continuous transmission mode and is now verified even for the DTx mode. However, which is quite surprising is that the results of NOMA system with optimal power allocation are very close to those of NOMA system with a power allocation where the transmit power is equal to the maximum transmit power (with this power allocation, the problem of (7) is solved considering that the first constraint, on the maximum transmit power, is and equality).

In Fig. 3, we examine the power consumption of the base 
station as a function of the average number of users in the cell coverage for a minimum capacity constraint fixed to 1 Mbits/s. Similarly, the results for NOMA technique when using DTx are compared to those of TDMA technique. We can notice that, regardless the average number of users in the cell coverage, the minimum power consumption is reached for NOMA technique.

\section{Conclusion}

In this paper, we have addressed the problem of power allocation with Cell DTx for minimizing the power consumption of a NOMA base station. We employed the KKT conditions so as to propose an algorithm that optimally solve this problem. We have shown that NOMA always benefits to the system with respect to TDMA in terms of power savings.

\section{References}

[1] Y. Saito, Y. Kishiyama, A. Benjebbour, T. Nakamura, A. Li, and K. Higuchi. Non-Orthogonal Multiple Access (NOMA) for Cellular Future Radio Access. In 2013 IEEE 77th Vehicular Technology Conference (VTC Spring), pages 1-5, June 2013.

[2] P. Frenger and al. Reducing energy consumption in lte with cell dtx. In Vehicular Technology Conference (VTC Spring), 2011 IEEE 73rd, pages 1-5, May 2011.
[3] H. Holtkamp, G. Auer, and H. Haas. On Minimizing Base Station Power Consumption. In 2011 IEEE Vehicular Technology Conference (VTC Fall), pages 1-5, Sept 2011.

[4] R. Bonnefoi, C. Moy, and J. Palicot. New macrocell downlink energy consumption minimization with cell $\mathrm{dtx}$ and power control. In 2017 IEEE International Conference on Communications (ICC), pages 1-7, May 2017.

[5] H. B. Ren, M. Zhao, J. K. Zhu, and W. Y. Zhou. Energyefficient resource allocation for OFDMA networks with sleep mode. Electronics Letters, 49(2):111-113, January 2013.

[6] R. Bonnefoi, C. Moy, H. Farès, and J. Palicot. Power allocation for minimizing energy consumption of ofdma downlink with cell dtx. In 2017 24th International Conference on Telecommunications (ICT), pages 1-6, May 2017.

[7] G. Auer and al. How much energy is needed to run a wireless network? Wireless Communications, IEEE, 18(5):40-49, October 2011.

[8] Z. Ding, Y. Liu, J. Choi, Q. Sun, M. Elkashlan, C. L. I, and H. V. Poor. Application of Non-Orthogonal Multiple Access in LTE and 5G Networks. IEEE Communications Magazine, 55(2):185-191, February 2017.

[9] Y. Zhang, H. Wang, Q. Yang, and Z. Ding. Secrecy sum rate maximization in non-orthogonal multiple access. IEEE Communications Letters, 20(5):930-933, May 2016. 